\title{
The Investigation of Exact Solutions for the Appropriate Type of the Dispersive Long Wave Equation
}

\author{
J. Biazar, M. B. Mehrlatifan, and Z. Salehdirin \\ Department of Applied Mathematics, Faculty of Mathematical Sciences, University of Guilan, Rasht 41335-19141, Iran \\ Correspondence should be addressed to M. B. Mehrlatifan; mehrlatifan@gmail.com
}

Received 27 November 2013; Accepted 1 February 2014; Published 19 March 2014

Academic Editors: G. Cleaver, J. Garecki, D. Gepner, R. Parwani, and G. F. Torres del Castillo

Copyright (C) 2014 J. Biazar et al. This is an open access article distributed under the Creative Commons Attribution License, which permits unrestricted use, distribution, and reproduction in any medium, provided the original work is properly cited.

Improved $\left(G^{\prime} / G\right)$-expansion and first integral methods are used to construct exact solutions of the $(2+1)$-dimensional Eckhaustype extension of the dispersive long wave equation. The $\left(G^{\prime} / G\right)$-expansion method is based on the assumptions that the travelling wave solutions can be expressed by a polynomial in $\left(G^{\prime} / G\right)$ and the first integral method is based on the theory of commutative algebra in which Division Theorem is of concern. It is worth mentioning that these methods are used for different systems and those two different systems can both be reduced to a system that will be mentioned in this paper. To recapitulate, this investigation has resulted in the exact solutions of the given systems.

\section{Introduction}

The investigation of exact solutions to nonlinear evolution has become an interesting subject in nonlinear science field, since the time when the soliton concept was first introduced by Zabusky and Kruskal in 1965 [1]. It was not until the mid1960s when applied scientists began to use modern digital computers to study nonlinear wave propagation that the soundness of Russell's early ideas began to be appreciated. He viewed the solitary wave as a self-sufficient dynamic entity; a "thing" displaying many properties of a particle. From the modern perspective it is used as a constructive element to formulate the complex dynamical behavior of wave systems throughout science: from hydrodynamics to nonlinear optics, from plasmas to shock waves, and from the elementary particles of matter to the elementary particles of thought. For a more detailed and technical account of the solitary wave, see [2].

In recent years, other methods have been developed, such as the Backlund transformation method [3], Darboux transformation [4], tanh method [5-7], extended tanh function method [8], the generalized hyperbolic function [9], and variable separation method [10].
The celebrated $(1+1)$-dimensional dispersive long wave equation $[11,12]$

$$
\begin{gathered}
u_{t}+v_{x}+\frac{1}{2}\left(u^{2}\right)_{x}=0, \\
v_{t}+\left(u v+u+u_{x x}\right)_{x}=0
\end{gathered}
$$

plays an important role in nonlinear physics; many properties of (1) have been reported [12,13]. It is interesting to study the extensions of (1) in higher-dimensional spaces. To date, there exist two prototypical of (1) to cover the situation of wide channel or open seas. In 1987, Boiti et al. [14] presented the following $(2+1)$-dimensional extension related to (1):

$$
\begin{aligned}
& u_{t y}+v_{x x}+\frac{1}{2}\left(u^{2}\right)_{x y}=0, \\
& v_{t}+\left(u v+u+u_{x y}\right)_{x}=0 .
\end{aligned}
$$

For (2), the Backlund transformation, soliton solutions are given $[14,15]$. In 1985, Eckhaus [16] presented another different two-dimensional extension of (1):

$$
\begin{gathered}
u_{t}+v_{x}+\frac{1}{2}\left(u^{2}\right)_{x}=0, \\
v_{t x}+\left(u v+u+u_{x x}\right)_{x x}+u_{y y}=0
\end{gathered}
$$


which was obtained in the appropriate approximation from the basic equations of hydrodynamics. It is easy to see that if one makes the transformation $u=u(x+y, t), v=v(x+y, t)-1$ then (3) can also be reduced to (1) so in the same way, (2) can also be reduced to (1). But as Boiti et al. [14] pointed out, system (2) is different from system (3). Therefore, the aim of this paper is to find exact solutions of the $(2+1)$-dimensional Eckhaus-type (2) and (3) by various methods.

\section{Methodology}

Consider a general nonlinear partial differential equation in the form

$$
F\left(u, u_{t}, u_{x}, u_{y}, u_{t t}, u_{x x}, u_{x t}, u_{x y}, \ldots\right)=0
$$

where $u=u(x, y, t)$ is the solution of nonlinear PDE Equation (4). Furthermore, the transformations which are used are as follows:

$$
u(t, x)=U(\xi), \quad \xi=\alpha(x+\beta y-\gamma t)
$$

where $\alpha, \beta$, and $\gamma$ are constants. Using the chain rule, it can be found that

$$
\begin{gathered}
\frac{\partial}{\partial t}(\cdot)=-\alpha \gamma \frac{\partial}{\partial \xi}(\cdot), \quad \frac{\partial}{\partial x}(\cdot)=\alpha \frac{\partial}{\partial \xi}(\cdot), \\
\frac{\partial^{2}}{\partial x^{2}}(\cdot)=\alpha^{2} \frac{\partial^{2}}{\partial \xi^{2}}(\cdot), \ldots
\end{gathered}
$$

At present, (6) is employed to change the nonlinear PDE Equation (4) to nonlinear ordinary differential equation

$$
G\left(U(\xi), U_{\xi}(\xi), U_{\xi \xi}(\xi), \ldots\right)=0
$$

2.1. The Improved $\left(G^{\prime} / G\right)$-Expansion Method. We initially predict the structure of the solution $U=U(\xi)$ to (7) in the finite series form

$$
U(\xi)=\sum_{i=0}^{m} a_{i}\left(\frac{G^{\prime}}{G}\right)^{i}+\sum_{j=1}^{m} b_{j}\left(\frac{G^{\prime}}{G}\right)^{-i},
$$

where $G=G(\xi)$ satisfies the second order LODE in the form

$$
G^{\prime \prime}+\lambda G+\mu G=0
$$

$a_{i}, b_{j},(i, j=0,1,2, \ldots, j \neq 0), \lambda$, and $\mu$ are constants to be determined later, and $a_{m} \neq 0$ or $b_{m} \neq 0$; the unwritten part in (8) is also a polynomial in $\left(G^{\prime} / G\right)$, but for the degree which is generally equal to or less than $m-1$, the positive integer $m$ can be determined by considering the homogeneous balance between the highest order derivatives and nonlinear terms appearing in ODE (7). Substituting (8) into (2) yields a system of nonlinear algebraic equations for $a_{i}, b_{j},(i, j=0,1,2, \ldots$, $j \neq 0), \lambda, \mu, \alpha, \beta$, and $\gamma$. Finally, substitution of the systems solutions into (8) gives traveling wave solutions to (1).
The general solutions of the second order LODE (9) have been well known for

(i) if $\lambda^{2}-4 \mu>0$,

$$
\begin{aligned}
\frac{G^{\prime}}{G}= & -\frac{\lambda}{2} \\
& +\frac{1}{2} \sqrt{\lambda^{2}-4 \mu} \\
& \times\left[\left(c_{1} \sinh \left(\frac{1}{2} \sqrt{\lambda^{2}-4 \mu \xi}\right)\right.\right. \\
& \left.+c_{2} \cosh \left(\frac{1}{2} \sqrt{\lambda^{2}-4 \mu} \xi\right)\right) \\
& \times\left(c_{1} \cosh \left(\frac{1}{2} \sqrt{\lambda^{2}-4 \mu} \xi\right)\right. \\
& \left.\left.\quad+c_{2} \sinh \left(\frac{1}{2} \sqrt{\lambda^{2}-4 \mu} \xi\right)\right)^{-1}\right],
\end{aligned}
$$

(ii) if $\lambda^{2}-4 \mu=0$,

$$
\frac{G^{\prime}}{G}=-\frac{\lambda}{2}+\frac{c_{2}}{c_{1}+c_{2} \xi},
$$

(iii) if $\lambda^{2}-4 \mu<0$,

$$
\begin{aligned}
\frac{G^{\prime}}{G}= & -\frac{\lambda}{2} \\
+ & \frac{1}{2} \sqrt{4 \mu-\lambda^{2}} \\
\times & {\left[\left(-c_{1} \sin \left(\frac{1}{2} \sqrt{4 \mu-\lambda^{2}} \xi\right)\right.\right.} \\
& \left.+c_{2} \cos \left(\frac{1}{2} \sqrt{4 \mu-\lambda^{2}} \xi\right)\right) \\
& \times\left(c_{1} \cos \left(\frac{1}{2} \sqrt{4 \mu-\lambda^{2}} \xi\right)\right. \\
& \left.\left.+c_{2} \sin \left(\frac{1}{2} \sqrt{4 \mu-\lambda^{2}} \xi\right)\right)^{-1}\right],
\end{aligned}
$$

where $c_{1}$ and $c_{2}$ are arbitrary constants.

2.2. The First Integral Method. By focusing on (4), a new independent variable is introduced as

$$
X(\xi)=u(\xi), \quad Y=\frac{\partial u(\xi)}{\partial \xi} .
$$

This yields a system of nonlinear ODEs

$$
X_{\xi}(\xi)=Y(\xi), \quad Y_{\xi}(\xi)=F_{1}(X(\xi),(\xi)) .
$$

If it is revealed that the integrals to (14) are under the same conditions of the qualitative theory of ordinary differential equation [17], then general solutions to (14) can be solved 
directly. However, it is generally so difficult for us to realize this even for one first integral. That is why for a given plane autonomous system there is no systematic theory that can tell us how to find its first integrals, nor is there a logical way for telling us what these first integrals are. Thus, Division Theorem is used to obtain one first integral of (14). Now, let us recall the Division Theorem.

Division Theorem. Suppose that $P(w, z)$ and $q(w, z)$ are polynomials in $C[w, z]$ and $P(w, z)$ is irreducible to $C[w, z]$. If $q(w, z)$ vanishes through all zero points of $P(w, z)$, then there exists a polynomial $G(w, z)$ in $C(w, z)$ such that $q(w, z)=$ $P(w, z) G(w, z)$. See [18].

\section{Application}

In this paper, improved $\left(G^{\prime} / G\right)$-expansion method is employed to study the solutions of the nonlinear partial differential system (2); afterwards, the first integral method is employed to study the solutions of the nonlinear partial differential system (3).

3.1. Explicit and Exact Solution of the System (2). We introduce the transformations,

$$
\begin{gathered}
u(x, y, t)=U(\xi), \quad v(x, y, t)=V(\xi), \\
\xi=\alpha(x+\beta y-\gamma t) .
\end{gathered}
$$

Substituting (15) into (2), there will be a change as follows:

$$
\begin{gathered}
-\beta \gamma U_{\xi \xi}(\xi)+V_{\xi \xi}(\xi)+\frac{1}{2} \beta\left(U^{2}(\xi)\right)_{\xi \xi}=0, \\
-\gamma V_{\xi}(\xi)+\left(U(\xi) V(\xi)+U+\alpha^{2} \beta U_{\xi \xi}(\xi)\right)_{\xi}=0,
\end{gathered}
$$

where by twice integrating the first equation of (16), in respect to $\xi$, it can be found that

$$
V(\xi)=\beta \gamma U(\xi)-\frac{1}{2} \beta U^{2}(\xi)+r_{1} .
$$

By integrating the second equation of (16), in respect to $\xi$, then substituting (17) into this equation, it can be found that

$$
\begin{aligned}
\alpha^{2} \beta U_{\xi \xi}(\xi) & +\frac{3}{2} \beta \gamma U^{2}(\xi)-\frac{1}{2} \beta U^{3}(\xi) \\
& +\left(r_{1}-\beta \gamma^{2}+1\right) U(\xi)+\left(\gamma r_{1}+r_{2}\right)=0,
\end{aligned}
$$

where $r_{1}$ and $r_{2}$ are arbitrary integration constants that are to be determined later.

Suppose that the solution of ODE (18) can be expressed by a polynomial in $\left(G^{\prime} / G\right)$ as follows:

$$
U(\xi)=\sum_{i=0}^{m} a_{i}\left(\frac{G^{\prime}}{G}\right)^{i}+\sum_{i=1}^{m} b_{i}\left(\frac{G^{\prime}}{G}\right)^{-i},
$$

where $G=G(\xi)$ satisfies the second order LODE (9). By balancing the term $U_{\xi \xi}$ with the term $U^{3}(\xi)$ in (18), we obtain $m=1$, so we can write (19) as

$$
U(\xi)=a_{0}+a_{1}\left(\frac{G^{\prime}}{G}\right)+b_{1}\left(\frac{G^{\prime}}{G}\right)^{-1} .
$$

And therefore

$$
\begin{aligned}
U^{3}(\xi)= & a_{0}^{3}+6 a_{0} a_{1} b_{1}+\left(3 a_{0}^{2} a_{1}+3 a_{1}^{2} b_{1}\right)\left(\frac{G^{\prime}}{G}\right) \\
& +3 a_{0} a_{1}^{2}\left(\frac{G^{\prime}}{G}\right)^{2}+a_{1}^{3}\left(\frac{G^{\prime}}{G}\right)^{3} \\
& +\left(3 a_{0}^{2} b_{1}+3 b_{1}^{2} a_{1}\right)\left(\frac{G^{\prime}}{G}\right)^{-1} \\
& +3 a_{0} b_{1}^{2}\left(\frac{G^{\prime}}{G}\right)^{-2}+b_{1}^{3}\left(\frac{G^{\prime}}{G}\right)^{-3}, \\
U_{\xi \xi}(\xi)= & a_{1} \lambda \mu+b_{1} \lambda+\left(a_{1} \lambda^{2}+2 a_{1} \mu\right)\left(\frac{G^{\prime}}{G}\right) \\
& +3 a_{1} \lambda\left(\frac{G^{\prime}}{G}\right)^{2}+2 a_{1}\left(\frac{G^{\prime}}{G}\right)^{3} \\
& +\left(2 b_{1} \mu+b_{1} \lambda^{2}\right)\left(\frac{G^{\prime}}{G}\right)^{-1} \\
& +3 b_{1} \lambda \mu\left(\frac{G^{\prime}}{G}\right)^{-2}+2 b_{1} \mu^{2}\left(\frac{G^{\prime}}{G}\right)^{-3} .
\end{aligned}
$$

By substituting (20) and (21) into ODE (18) and collecting all terms with the same power of $\left(G^{\prime} / G\right)$ together, the lefthand side of ODE (18) is converted into another polynomial in $\left(G^{\prime} / G\right)$. Equating each coefficient of this polynomial to zero yields a set of simultaneous algebraic equations for $a_{0}, a_{1}, b_{1}$, $r_{1}, r_{2}, \lambda$, and $\mu$. Having solved the given equation with aid Maple, the following solutions will be attained:

$$
\begin{gathered}
a_{0}= \pm a_{0}, \quad a_{1}=0, \\
b_{1}= \pm 2 \alpha \mu, \quad r_{1}=\frac{1}{2} a_{0}^{2} \beta-\beta \gamma a_{0}-2 \alpha^{2} \beta \mu-1, \\
\lambda=\lambda, \quad \mu=\mu, \\
r_{2}=2 \beta \gamma^{2} a_{0}-\beta \gamma a_{0}^{2}+4 \alpha^{2} \beta \mu \gamma+\gamma, \\
a_{0}=a_{0}, \quad a_{1}= \pm 2 \alpha, \\
b_{1}=0, \quad r_{1}=\frac{1}{2} a_{0}^{2} \beta-\beta \gamma a_{0}-2 \alpha^{2} \beta \mu-1, \\
\lambda=\mp \frac{\gamma-a_{0}}{\alpha}, \quad \mu=\mu, \\
r_{2}=2 \beta \gamma^{2} a_{0}-\beta \gamma a_{0}^{2}+4 \alpha^{2} \beta \mu \gamma+\gamma, \\
a_{0}=a_{0}, \quad a_{1}= \pm 2 \alpha, \\
r_{1}=-\frac{1}{2} a_{0}^{2} \beta-\beta \gamma a_{0} \pm 2 \alpha \beta b_{1}-1, \\
\lambda=\mp \frac{\gamma-a_{0}}{\alpha}, \quad \mu= \pm \frac{b_{1}}{2 \alpha}, \\
b_{1}=b_{1}, \quad \begin{array}{c}
\alpha \beta \gamma b_{1}+2 \beta \gamma^{2} a_{0}-\beta \gamma a_{0}^{2}+2 a_{0} \alpha \beta b_{1}+\gamma,
\end{array}
\end{gathered}
$$




$$
\begin{aligned}
& a_{0}= \pm \alpha \lambda+\gamma, \quad a_{1}= \pm 2 \alpha, \\
& b_{1}=0, \quad r_{1}=\frac{1}{2} \alpha^{2} \beta \lambda^{2}-\frac{1}{2} \beta \gamma^{2}-2 \alpha^{2} \beta \mu-1 \text {, } \\
& \lambda=\lambda, \quad \mu=\mu, \\
& r_{2}=\beta \gamma^{3}+\gamma-\alpha^{2} \beta \lambda^{2} \gamma+4 \alpha^{2} \beta \mu \gamma, \\
& a_{0}= \pm \alpha \lambda+\gamma, \quad a_{1}=0, \\
& b_{1}= \pm 2 \alpha \mu, \quad r_{1}=\frac{1}{2} \alpha^{2} \beta \lambda^{2}-\frac{1}{2} \beta \gamma^{2}-2 \alpha^{2} \beta \mu-1 \text {, } \\
& \lambda=\lambda, \quad \mu=\mu, \\
& r_{2}=\beta \gamma^{3}+\gamma-\alpha^{2} \beta \lambda^{2} \gamma+4 \alpha^{2} \beta \mu \gamma .
\end{aligned}
$$

By using (22)-(26), expression (20) can be written as follows:

$$
\begin{gathered}
U(\xi)= \pm a_{0} \pm 2 \alpha \mu\left(\frac{G^{\prime}}{G}\right)^{-1}, \\
U(\xi)=a_{0} \pm 2 \alpha\left(\frac{G^{\prime}}{G}\right), \\
U(\xi)=a_{0} \pm 2 \alpha\left(\frac{G^{\prime}}{G}\right)+b_{1}\left(\frac{G^{\prime}}{G}\right)^{-1}, \\
U(\xi)=( \pm \alpha \lambda+\gamma) \pm 2 \alpha\left(\frac{G^{\prime}}{G}\right), \\
U(\xi)=( \pm \alpha \lambda+\gamma) \pm 2 \alpha \mu\left(\frac{G^{\prime}}{G}\right)^{-1}
\end{gathered}
$$

Equations (27)-(31) are the formula of solution of (18). Substituting the general solutions of (9) into (27)-(31) we have three types of travelling wave solutions of the $(2+1)$ dimensional Eckhaus-type equation (2) (so $u_{i j}$ is definiendum $i$ th type solution from $j$ th expression) as follows.

Case A. When $\lambda^{2}-4 \mu>0$,

$$
\begin{aligned}
& u_{1^{28}}(x, y, t) \\
& = \pm a_{0} \pm 4 \alpha \mu \\
& \quad \times\left(\sqrt{\lambda^{2}-4 \mu}\right. \\
& \times\left(\left(c _ { 1 } \operatorname { s i n h } \left(\frac{1}{2} \alpha \sqrt{\lambda^{2}-4 \mu}\right.\right.\right. \\
& \quad \times(x+\beta y-\gamma t)) \\
& +c_{2} \cosh \left(\frac{1}{2} \alpha \sqrt{\lambda^{2}-4 \mu}\right. \\
& \quad \times(x+\beta y-\gamma t)))
\end{aligned}
$$

$$
\begin{aligned}
\times\left(c _ { 2 } \operatorname { s i n h } \left(\frac{1}{2} \alpha \sqrt{\lambda^{2}-4 \mu}\right.\right. & \\
& \times(x+\beta y-\gamma t)) \\
+c_{1} \cosh \left(\frac{1}{2} \sqrt{\lambda^{2}-4 \mu}\right. & \left.\times(x+\beta y-\gamma t)))^{-1}\right) \\
& \\
-\lambda)^{-1}, &
\end{aligned}
$$$$
v_{1^{28}}(x, y, t)
$$$$
=\beta \gamma u_{1^{28}}(x, y, t)-\frac{1}{2} \beta\left(u_{1^{28}}(x, y, t)\right)^{2}
$$$$
+\frac{1}{2} a_{0}^{2} \beta-\beta \gamma a_{0}-2 \alpha^{2} \beta \mu-1
$$$$
u_{1^{29}}(x, y, t)
$$$$
=a_{0} \pm \alpha
$$$$
\times\left(\sqrt{\lambda^{2}-4 \mu}\right.
$$$$
\times\left(\left(c _ { 1 } \operatorname { s i n h } \left(\frac{1}{2} \alpha \sqrt{\lambda^{2}-4 \mu}\right.\right.\right.
$$$$
\times(x+\beta y-\gamma t))
$$$$
+c_{2} \cosh \left(\frac{1}{2} \alpha \sqrt{\lambda^{2}-4 \mu}\right.
$$$$
\times(x+\beta y-\gamma t)))
$$$$
\times\left(c _ { 2 } \operatorname { s i n h } \left(\frac{1}{2} \alpha \sqrt{\lambda^{2}-4 \mu}\right.\right.
$$$$
\times(x+\beta y-\gamma t))
$$$$
+c_{1} \cosh \left(\frac{1}{2} \sqrt{\lambda^{2}-4 \mu}\right.
$$$$
\left.\times(x+\beta y-\gamma t)))^{-1}\right)
$$

$-\lambda)$

$v_{1^{29}}(x, y, t)$

$$
\begin{aligned}
= & \beta \gamma u_{1^{29}}(x, y, t)-\frac{1}{2} \beta\left(u_{1^{29}}(x, y, t)\right)^{2} \\
& +\frac{1}{2} a_{0}^{2} \beta-\beta \gamma a_{0}-2 \alpha^{2} \beta \mu-1,
\end{aligned}
$$




$$
\begin{aligned}
& u_{1^{30}}(x, y, t) \\
& =a_{0} \pm \alpha \\
& \times\left(\sqrt{\lambda^{2}-4 \mu}\right. \\
& \times\left(\left(c _ { 1 } \operatorname { s i n h } \left(\frac{1}{2} \alpha \sqrt{\lambda^{2}-4 \mu}\right.\right.\right. \\
& \times(x+\beta y-\gamma t)) \\
& +c_{2} \cosh \left(\frac{1}{2} \alpha \sqrt{\lambda^{2}-4 \mu}\right. \\
& \times(x+\beta y-\gamma t))) \\
& \times\left(c _ { 2 } \operatorname { s i n h } \left(\frac{1}{2} \alpha \sqrt{\lambda^{2}-4 \mu}\right.\right. \\
& \times(x+\beta y-\gamma t)) \\
& +c_{1} \cosh \left(\frac{1}{2} \sqrt{\lambda^{2}-4 \mu}\right. \\
& \left.\times(x+\beta y-\gamma t)))^{-1}\right) \\
& -\lambda)+2 b_{1} \\
& \times\left(\sqrt{\lambda^{2}-4 \mu}\right. \\
& \times\left(\left(c _ { 1 } \operatorname { s i n h } \left(\frac{1}{2} \alpha \sqrt{\lambda^{2}-4 \mu}\right.\right.\right. \\
& \times(x+\beta y-\gamma t)) \\
& +c_{2} \cosh \left(\frac{1}{2} \alpha \sqrt{\lambda^{2}-4 \mu}\right. \\
& \times(x+\beta y-\gamma t))) \\
& \times\left(\left(c _ { 2 } \operatorname { s i n h } \left(\frac{1}{2} \alpha \sqrt{\lambda^{2}-4 \mu}\right.\right.\right. \\
& \times(x+\beta y-\gamma t)) \\
& +c_{1} \cosh \left(\frac{1}{2} \sqrt{\lambda^{2}-4 \mu}\right. \\
& \left.\times(x+\beta y-\gamma t)))^{-1}\right) \\
& v_{1^{30}}(x, y, t) \\
& =\beta \gamma u_{1^{30}}(x, y, t)-\frac{1}{2} \beta\left(u_{1^{30}}(x, y, t)\right)^{2} \\
& -\frac{1}{2} a_{0}^{2} \beta-\beta \gamma a_{0} \pm 2 \alpha \beta b_{1}-1, \\
& u_{1^{31}}(x, y, t) \\
& =( \pm \alpha \lambda+\gamma) \pm \alpha \\
& \times\left(\sqrt{\lambda^{2}-4 \mu}\right. \\
& \times\left(\left(c _ { 1 } \operatorname { s i n h } \left(\frac{1}{2} \alpha \sqrt{\lambda^{2}-4 \mu}\right.\right.\right. \\
& \times(x+\beta y-\gamma t)) \\
& +c_{2} \cosh \left(\frac{1}{2} \alpha \sqrt{\lambda^{2}-4 \mu}\right. \\
& \times(x+\beta y-\gamma t))) \\
& \times\left(c _ { 2 } \operatorname { s i n h } \left(\frac{1}{2} \alpha \sqrt{\lambda^{2}-4 \mu}\right.\right. \\
& \times(x+\beta y-\gamma t)) \\
& +c_{1} \cosh \left(\frac{1}{2} \sqrt{\lambda^{2}-4 \mu}\right. \\
& \left.\times(x+\beta y-\gamma t)))^{-1}\right) \\
& -\lambda) \\
& v_{1^{31}}(x, y, t) \\
& =\beta \gamma u_{1^{31}}(x, y, t)-\frac{1}{2} \beta\left(u_{1^{31}}(x, y, t)\right)^{2} \\
& +\frac{1}{2} \alpha^{2} \beta \lambda^{2}-\frac{1}{2} \beta \gamma^{2}-2 \alpha^{2} \beta \mu-1, \\
& u_{1^{32}}(x, y, t) \\
& =( \pm \alpha \lambda+\gamma) \pm 4 \alpha \mu \\
& \times\left(\sqrt{\lambda^{2}-4 \mu}\right. \\
& \times\left(\left(c _ { 1 } \operatorname { s i n h } \left(\frac{1}{2} \alpha \sqrt{\lambda^{2}-4 \mu}\right.\right.\right. \\
& \times(x+\beta y-\gamma t)) \\
& +c_{2} \cosh \left(\frac{1}{2} \alpha \sqrt{\lambda^{2}-4 \mu}\right. \\
& -\lambda)^{-1} \\
& \times(x+\beta y-\gamma t)))
\end{aligned}
$$




$$
\begin{gathered}
\times\left(c _ { 2 } \operatorname { s i n h } \left(\frac{1}{2} \alpha \sqrt{\lambda^{2}-4 \mu}\right.\right. \\
\times(x+\beta y-\gamma t)) \\
+c_{1} \cosh \left(\frac{1}{2} \sqrt{\lambda^{2}-4 \mu}\right. \\
\left.\times(x+\beta y-\gamma t)))^{-1}\right) \\
-\lambda)^{-1}, \quad \frac{1}{v_{132}(x, y, t)} \\
=\beta \gamma u_{1^{32}}(x, y, t)-\frac{1}{2} \beta\left(u_{1^{32}}(x, y, t)\right)^{2} \\
+\frac{1}{2} \alpha^{2} \beta \lambda^{2}-\frac{1}{2} \beta \gamma^{2}-2 \alpha^{2} \beta \mu-1,
\end{gathered}
$$

$$
\begin{aligned}
& u_{2^{29}}(x, y, t) \\
& =a_{0} \pm \alpha \\
& \times\left(\sqrt{4 \mu-\lambda^{2}}\right. \\
& \times\left(\left(-c_{1} \sin \left(\frac{1}{2} \alpha \sqrt{4 \mu-\lambda^{2}}\right.\right.\right. \\
& \times(x+\beta y-\gamma t)) \\
& +c_{2} \cos \left(\frac{1}{2} \alpha \sqrt{4 \mu-\lambda^{2}}\right. \\
& \times(x+\beta y-\gamma t))) \\
& \times\left(c _ { 2 } \operatorname { s i n } \left(\frac{1}{2} \alpha \sqrt{4 \mu-\lambda^{2}}\right.\right. \\
& \times(x+\beta y-\gamma t)) \\
& +c_{1} \cos \left(\frac{1}{2} \sqrt{4 \mu-\lambda^{2}}\right. \\
& \left.\times(x+\beta y-\gamma t)))^{-1}\right) \\
& -\lambda)
\end{aligned}
$$

$v_{2^{29}}(x, y, t)$

$$
\begin{gathered}
=\beta \gamma u_{2^{29}}(x, y, t)-\frac{1}{2} \beta\left(u_{2^{29}}(x, y, t)\right)^{2} \\
+\frac{1}{2} \alpha^{2} \beta \lambda^{2}-\frac{1}{2} \beta \gamma^{2}-2 \alpha^{2} \beta \mu-1,
\end{gathered}
$$$$
u_{2^{30}}(x, y, t)
$$$$
=a_{0} \pm \alpha
$$$$
\times\left(c _ { 2 } \operatorname { s i n } \left(\frac{1}{2} \alpha \sqrt{4 \mu-\lambda^{2}}\right.\right.
$$$$
\times(x+\beta y-\gamma t))
$$$$
+c_{1} \cos \left(\frac{1}{2} \sqrt{4 \mu-\lambda^{2}}\right.
$$$$
\left.\times(x+\beta y-\gamma t)))^{-1}\right)
$$$$
\times\left(\sqrt{4 \mu-\lambda^{2}}\right.
$$$$
\times\left(\left(-c_{1} \sin \left(\frac{1}{2} \alpha \sqrt{4 \mu-\lambda^{2}}\right.\right.\right.
$$$$
\times(x+\beta y-\gamma t))
$$$$
+c_{2} \cos \left(\frac{1}{2} \alpha \sqrt{4 \mu-\lambda^{2}}\right.
$$$$
\times(x+\beta y-\gamma t)))
$$

$$
v_{2^{28}}(x, y, t)
$$$$
=\beta \gamma u_{2^{28}}(x, y, t)-\frac{1}{2} \beta\left(u_{2^{28}}(x, y, t)\right)^{2}
$$$$
\times\left(c _ { 2 } \operatorname { s i n } \left(\frac{1}{2} \alpha \sqrt{4 \mu-\lambda^{2}}\right.\right.
$$$$
+\frac{1}{2} \alpha^{2} \beta \lambda^{2}-\frac{1}{2} \beta \gamma^{2}-2 \alpha^{2} \beta \mu-1,
$$

$$
\times(x+\beta y-\gamma t))
$$




$$
\begin{aligned}
& +c_{1} \cos \left(\frac{1}{2} \sqrt{4 \mu-\lambda^{2}}\right. \\
& \left.\times(x+\beta y-\gamma t)))^{-1}\right) \\
& -\lambda)+2 b_{1} \\
& \times\left(\sqrt{4 \mu-\lambda^{2}}\right. \\
& \times\left(\left(-c_{1} \sin \left(\frac{1}{2} \alpha \sqrt{4 \mu-\lambda^{2}}\right.\right.\right. \\
& \times(x+\beta y-\gamma t)) \\
& +c_{2} \cos \left(\frac{1}{2} \alpha \sqrt{4 \mu-\lambda^{2}}\right. \\
& \times(x+\beta y-\gamma t))) \\
& \times\left(c _ { 2 } \operatorname { s i n } \left(\frac{1}{2} \alpha \sqrt{4 \mu-\lambda^{2}}\right.\right. \\
& \times(x+\beta y-\gamma t)) \\
& +c_{1} \cos \left(\frac{1}{2} \sqrt{4 \mu-\lambda^{2}}\right. \\
& \left.\times(x+\beta y-\gamma t)))^{-1}\right) \\
& -\lambda)^{-1}
\end{aligned}
$$$$
v_{2^{30}}(x, y, t)
$$$$
=\beta \gamma u_{2^{30}}(x, y, t)-\frac{1}{2} \beta\left(u_{2^{30}}(x, y, t)\right)^{2}
$$$$
+\frac{1}{2} \alpha^{2} \beta \lambda^{2}-\frac{1}{2} \beta \gamma^{2}-2 \alpha^{2} \beta \mu-1,
$$$$
u_{2^{31}}(x, y, t)
$$$$
=( \pm \alpha \lambda+\gamma) \pm \alpha
$$$$
\times\left(\sqrt{4 \mu-\lambda^{2}}\right.
$$$$
\times\left(\left(-c_{1} \sin \left(\frac{1}{2} \alpha \sqrt{4 \mu-\lambda^{2}}\right.\right.\right.
$$$$
\times(x+\beta y-\gamma t))
$$$$
+c_{2} \cos \left(\frac{1}{2} \alpha \sqrt{4 \mu-\lambda^{2}}\right.
$$$$
\times(x+\beta y-\gamma t)))
$$

$$
\begin{aligned}
\times\left(c _ { 2 } \operatorname { s i n } \left(\frac{1}{2} \alpha \sqrt{4 \mu-\lambda^{2}}\right.\right. \\
\times(x+\beta y-\gamma t)) \\
+c_{1} \cos \left(\frac{1}{2} \sqrt{4 \mu-\lambda^{2}}\right. \\
\left.\times(x+\beta y-\gamma t)))^{-1}\right)
\end{aligned}
$$

$-\lambda)$

$$
\begin{aligned}
& v_{2^{31}}(x, y, t) \\
& =\beta \gamma u_{2^{31}}(x, y, t)-\frac{1}{2} \beta\left(u_{2^{31}}(x, y, t)\right)^{2} \\
& \quad+\frac{1}{2} \alpha^{2} \beta \lambda^{2}-\frac{1}{2} \beta \gamma^{2}-2 \alpha^{2} \beta \mu-1,
\end{aligned}
$$$$
u_{2^{32}}(x, y, t)
$$$$
=( \pm \alpha \lambda+\gamma) \pm 4 \alpha \mu
$$$$
\times\left(\sqrt{4 \mu-\lambda^{2}}\right.
$$$$
\times\left(\left(-c_{1} \sin \left(\frac{1}{2} \alpha \sqrt{4 \mu-\lambda^{2}}\right.\right.\right.
$$$$
\times(x+\beta y-\gamma t))
$$$$
+c_{2} \cos \left(\frac{1}{2} \alpha \sqrt{4 \mu-\lambda^{2}}\right.
$$$$
\times(x+\beta y-\gamma t))
$$

$$
\begin{aligned}
\times\left(c _ { 2 } \operatorname { s i n } \left(\frac{1}{2} \alpha \sqrt{4 \mu-\lambda^{2}}\right.\right. \\
\times(x+\beta y-\gamma t)) \\
+c_{1} \cos \left(\frac{1}{2} \sqrt{4 \mu-\lambda^{2}}\right. \\
\left.\times(x+\beta y-\gamma t)))^{-1}\right)
\end{aligned}
$$

$$
-\lambda)^{-1}
$$

$v_{2^{32}}(x, y, t)$

$$
\begin{gathered}
=\beta \gamma u_{2^{32}}(x, y, t)-\frac{1}{2} \beta\left(u_{2^{32}}(x, y, t)\right)^{2} \\
+\frac{1}{2} \alpha^{2} \beta \lambda^{2}-\frac{1}{2} \beta \gamma^{2}-2 \alpha^{2} \beta \mu-1,
\end{gathered}
$$

where $c_{1}$ and $c_{2}$ are arbitrary constants. 
Case C. When $\lambda^{2}-4 \mu=0$,

$$
\begin{aligned}
u_{3^{28}}(x, y, t)= \pm a_{0} & \pm 4 \alpha \mu\left(\frac{2 c_{2}}{c_{1}+c_{2} \alpha(x+\beta y-\gamma t)}-\lambda\right)^{-1}, \\
v_{3^{28}}(x, y, t)= & \beta \gamma u_{3^{28}}(x, y, t)-\frac{1}{2} \beta\left(u_{3^{28}}(x, y, t)\right)^{2} \\
& +\frac{1}{2} \alpha^{2} \beta \lambda^{2}-\frac{1}{2} \beta \gamma^{2}-2 \alpha^{2} \beta \mu-1,
\end{aligned}
$$$$
u_{3^{29}}(x, y, t)=a_{0} \pm \alpha\left(\frac{2 c_{2}}{c_{1}+c_{2} \alpha(x+\beta y-\gamma t)}-\lambda\right)
$$$$
v_{3^{29}}(x, y, t)=\beta \gamma u_{3^{29}}(x, y, t)-\frac{1}{2} \beta\left(u_{3^{29}}(x, y, t)\right)^{2}
$$$$
+\frac{1}{2} \alpha^{2} \beta \lambda^{2}-\frac{1}{2} \beta \gamma^{2}-2 \alpha^{2} \beta \mu-1,
$$$$
u_{3^{30}}(x, y, t)=a_{0} \pm \alpha\left(\frac{2 c_{2}}{c_{1}+c_{2} \alpha(x+\beta y-\gamma t)}-\lambda\right)
$$$$
+2 b_{1}\left(\frac{2 c_{2}}{c_{1}+c_{2} \alpha(x+\beta y-\gamma t)}-\lambda\right)^{-1},
$$$$
v_{3^{30}}(x, y, t)=\beta \gamma u_{3^{30}}(x, y, t)-\frac{1}{2} \beta\left(u_{3^{30}}(x, y, t)\right)^{2}
$$$$
+\frac{1}{2} \alpha^{2} \beta \lambda^{2}-\frac{1}{2} \beta \gamma^{2}-2 \alpha^{2} \beta \mu-1,
$$$$
u_{3^{31}}(x, y, t)=( \pm \alpha \lambda+\gamma)
$$$$
\pm \alpha\left(\frac{2 c_{2}}{c_{1}+c_{2} \alpha(x+\beta y-\gamma t)}-\lambda\right),
$$$$
v_{3^{31}}(x, y, t)=\beta \gamma u_{3^{31}}(x, y, t)-\frac{1}{2} \beta\left(u_{3^{31}}(x, y, t)\right)^{2}
$$$$
+\frac{1}{2} \alpha^{2} \beta \lambda^{2}-\frac{1}{2} \beta \gamma^{2}-2 \alpha^{2} \beta \mu-1,
$$$$
u_{3^{32}}(x, y, t)=( \pm \alpha \lambda+\gamma)
$$$$
\pm 4 \alpha \mu\left(\frac{2 c_{2}}{c_{1}+c_{2} \alpha(x+\beta y-\gamma t)}-\lambda\right)^{-1},
$$$$
v_{3^{32}}(x, y, t)=\beta \gamma u_{3^{32}}(x, y, t)-\frac{1}{2} \beta\left(u_{3^{32}}(x, y, t)\right)^{2}
$$$$
+\frac{1}{2} \alpha^{2} \beta \lambda^{2}-\frac{1}{2} \beta \gamma^{2}-2 \alpha^{2} \beta \mu-1,
$$

where $c_{1}$ and $c_{2}$ are arbitrary constants. Using expression (28) in $u_{1^{29}}, u_{2^{29}}$, and $u_{3^{29}}$, it can be acquired that $\lambda= \pm\left(\gamma-a_{0}\right) / \alpha$ and by means of expression (29) in $u_{1^{30}}, u_{2^{30}}$, and $u_{3^{30}}$ one gets $\lambda=\mp\left(\gamma-a_{0}\right) / \alpha$ and $\mu=b_{1} / 2 \alpha$.
3.2. Explicit and Exact Solution of the System (3). Now, we look for solutions to (3) by the first integral method. By using (15) into (3) we can get

$$
\begin{gathered}
-\gamma U_{\xi}(\xi)+V_{\xi}(\xi)+\frac{1}{2}\left(U^{2}(\xi)\right)_{\xi}=0, \\
-\gamma V_{\xi \xi}(\xi)+\left(U(\xi) V(\xi)+U(\xi)+\alpha^{2} U_{\xi \xi}(\xi)\right)_{\xi \xi} \\
+\beta^{2} U_{\xi \xi}(\xi)=0,
\end{gathered}
$$

where by once integrating the first equation of (36), with respect to $\xi$, it can be found that

$$
V(\xi)=\gamma U(\xi)-\frac{1}{2} U^{2}(\xi)+r_{1} .
$$

By twice integrating the second equation of (16), with respect to $\xi$ and then substituting (37) into this equation, it can be found that

$$
\begin{aligned}
& \alpha^{2} U_{\xi \xi}(\xi)-\frac{1}{2} U^{3}(\xi)+\frac{3}{2} \gamma U^{2}(\xi) \\
& \quad+\left(r_{1}+\beta^{2}-\gamma^{2}+1\right) U(\xi)+\left(r_{2}-\gamma r_{1}\right)=0,
\end{aligned}
$$

where $r_{1}$ and $r_{2}$ are arbitrary integration constants that are to be determined later.

According to the first integral method, by using (13) and (14), it will be determined that

$$
\begin{gathered}
\dot{X}(\xi)=Y(\xi) \\
\dot{Y}(\xi)=\frac{1}{2 \alpha^{2}} U^{3}(\xi)-\frac{3}{2 \alpha^{2}} \gamma U^{2}(\xi) \\
+\left(\gamma^{2}-\beta^{2}-\left(r_{1}+1\right)\right) U(\xi)-\left(\frac{r_{2}-\gamma r_{1}}{\alpha^{2}}\right) .
\end{gathered}
$$

We suppose that $X(\xi)$ and $Y(\xi)$ are nontrivial solutions of (42) and $q[X, Y]=\sum_{i=0}^{m} a_{i}(X) Y^{i}=0$ is an irreducible polynomial in the complex domain $C[X, Y]$ such that

$$
q[X(\xi), Y(\xi)]=\sum_{i=0}^{m} a_{i}(X) Y^{i}=0
$$

where $a_{i}(X)(i=0, \ldots, m)$ are polynomials of $X$ and $a_{m}(X) \neq 0$. Due to Division Theorem, there exists a polynomial $g(X)+h(X) Y$ in the complex domain $C[X, Y]$, such that

$$
\begin{aligned}
\frac{d q}{d \xi} & =\frac{d q}{d X} \cdot \frac{d X}{d \xi}+\frac{d q}{d Y} \cdot \frac{d Y}{d \xi} \\
& =(g(X)+h(X) Y) \sum_{i=0}^{m} a_{i}(X) Y^{i} .
\end{aligned}
$$


By assuming that $m=3$ in (43) and by equating the coefficients of $Y^{i}(i=4,3,2,1,0)$ on both sides of (44), there will be

$$
\begin{aligned}
& \dot{a}_{3}(X)=a_{3}(X) h(X), \\
& \dot{a}_{2}(X)=a_{3}(X) g(X)+a_{2}(X) h(X), \\
& \dot{a}_{1}(X)=a_{2}(X) g(X) a_{1}(X) h(X) \\
& -3 a_{3}(X)\left(\frac{1}{2 \alpha^{2}} U^{3}(\xi)-\frac{3}{2 \alpha^{2}} \gamma U^{2}(\xi)\right. \\
& +\left(\gamma^{2}-\beta^{2}-\left(r_{1}+1\right)\right) U(\xi) \\
& \left.-\left(\frac{r_{2}-\gamma r_{1}}{\alpha^{2}}\right)\right) \\
& \dot{a}_{0}(X)=a_{1}(X) g(X)+a_{0}(X) h(X) \\
& -2 a_{2}(X)\left(\frac{1}{2 \alpha^{2}} U^{3}(\xi)-\frac{3}{2 \alpha^{2}} \gamma U^{2}(\xi)\right. \\
& +\left(\gamma^{2}-\beta^{2}-\left(r_{1}+1\right)\right) U(\xi) \\
& \left.-\left(\frac{r_{2}-\gamma r_{1}}{\alpha^{2}}\right)\right), \\
& a_{1}(X)\left(\frac{1}{2 \alpha^{2}} U^{3}(\xi)-\frac{3}{2 \alpha^{2}} \gamma U^{2}(\xi)\right. \\
& +\left(\gamma^{2}-\beta^{2}-\left(r_{1}+1\right)\right) U(\xi) \\
& \left.-\left(\frac{r_{2}-\gamma r_{1}}{\alpha^{2}}\right)\right) \\
& =a_{0}(X) g(X) \text {. }
\end{aligned}
$$

Since $a_{3}(X)$ is a polynomial of $X$, then, from (45), it may be deduced that $a_{3}(X)$ is a constant and $h(X)=0$, and we take $a_{3}(X)=1$. Balancing the degrees of $g(X), a_{2}(X), a_{1}(X)$, and $a_{0}(X)$, it is concluded that $\operatorname{deg} g(X)=1$, only.

Suppose that $g(X)=B_{0}+2 A_{1} X$ and $\left(A_{1} \neq 0\right)$ and then we will find that

$$
a_{2}(X)=A_{1} X^{2}+B_{0} X+A_{0},
$$

where $A_{0}$ is an arbitrary integration constant. Substituting $a_{0}(X), a_{1}(X), a_{2}(X)$, and $g(X)$ into (49) and setting all the coefficients of powers $X$ to be zero, then a system of nonlinear algebraic equations will be resulted. Having solved the given equation, the following solutions will be attained:

$$
\begin{gathered}
A_{1}=-\frac{1}{2 \alpha}, \quad A_{0}=A_{0}, \quad B_{0}=\frac{\gamma}{\alpha}, \\
d_{1}=d_{1}, \quad d_{2}=d_{1} A_{0}, \quad r_{1}=-\beta^{2}+\alpha A_{0}-1, \\
r_{2}=-\beta^{2} \gamma-\gamma, \\
A_{1}=\frac{1}{2 \alpha}, \quad A_{0}=0, \\
B_{0}=-\frac{\gamma}{\alpha}, \quad d_{1}=d_{2}=0, \quad r_{1}=-\beta^{2}-1,
\end{gathered}
$$

$$
\begin{gathered}
r_{2}=-\beta^{2} \gamma-\gamma, \\
A_{1}=\frac{3}{2 \alpha}, \quad A_{0}=A_{0}, \\
B_{0}=-\frac{3 \gamma}{\alpha}, \quad d_{1}=\frac{A_{0}^{2}}{3}, \quad d_{2}=\frac{A_{0}^{3}}{27}, \\
r_{1}=-\beta^{2}-\frac{1}{3} \alpha A_{0}-1, \quad r_{2}=-\beta^{2} \gamma-\gamma, \\
A_{1}=-\frac{3}{2 \alpha}, \quad A_{0}=A_{0}, \quad B_{0}=\frac{3 \gamma}{\alpha}, \\
d_{1}=\frac{A_{0}^{2}}{3}, \quad d_{2}=\frac{A_{0}^{3}}{27}, \quad r_{1}=-\beta^{2}+\frac{1}{3} \alpha A_{0}-1, \\
r_{2}=-\beta^{2} \gamma-\gamma,
\end{gathered}
$$

where $d_{1}$ and $d_{2}$ are arbitrary integration constants. Using the conditions (3.2) in (43), it can be searched out that

$$
\begin{gathered}
Y(\xi)=-\frac{1}{2}\left(\frac{-X^{2}+2 \gamma X+2 \alpha A_{0}}{\alpha}\right), \\
Y(\xi)= \pm \frac{1}{2}\left(\frac{X(2 \gamma-X)}{\alpha}\right), \\
Y(\xi)=\frac{1}{6}\left(\frac{-3 X^{2}+6 \gamma X-2 \alpha A_{0}}{\alpha}\right), \\
Y(\xi)=-\frac{1}{6}\left(\frac{-3 X^{2}+6 \gamma X+2 \alpha A_{0}}{\alpha}\right) .
\end{gathered}
$$

Expression (52) is the first integral of (42). Combining (52) with (42), the exact solution to system (3) will be found as follows:

$$
\begin{aligned}
& u_{1}(x, y, t) \\
& =\gamma-\left(\sqrt{2 \alpha A_{0}+\gamma^{2}}\right. \\
& \times \tanh \left(\frac{1}{2} \sqrt{2 \alpha A_{0}+\gamma^{2}}\right. \\
& \left.\left.\times\left((x+\beta y-\gamma t)+\xi_{0}\right)\right)\right), \\
& v_{1}(x, y, t)=\gamma u_{1}(x, y, t) \\
& -\frac{1}{2} u_{1}^{2}(x, y, t)-\beta^{2}+\alpha A_{0}-1, \\
& u_{2}(x, y, t)=\gamma \operatorname{sech}\left(\frac{\gamma}{2}\left((x+\beta y-\gamma t)+\xi_{0}\right)\right) \text {, } \\
& v_{2}(x, y, t)=\gamma u_{2}(x, y, t)-\frac{1}{2} u_{2}^{2}(x, y, t)-\beta^{2}-1,
\end{aligned}
$$




$$
\begin{aligned}
& u_{3}(x, y, t) \\
& =\gamma-\left(\frac{1}{3} \sqrt{6 \alpha A_{0}-9 \gamma^{2}}\right. \\
& \times \tanh \left(\frac{1}{2} \sqrt{6 \alpha A_{0}-9 \gamma^{2}}\right. \\
& \left.\left.\times\left((x+\beta y-\gamma t)+\xi_{0}\right)\right)\right), \\
& v_{3}(x, y, t)=\gamma u_{3}(x, y, t) \\
& -\frac{1}{2} u_{3}^{2}(x, y, t)-\beta^{2}-\frac{1}{3} \alpha A_{0}-1, \\
& u_{4}(x, y, t) \\
& =\gamma-\left(\frac{1}{3} \sqrt{6 \alpha A_{0}+9 \gamma^{2}}\right. \\
& \times \tanh \left(\frac{1}{2} \sqrt{6 \alpha A_{0}+9 \gamma^{2}}\right. \\
& \left.\left.\times\left((x+\beta y-\gamma t)+\xi_{0}\right)\right)\right) \\
& v_{4}(x, y, t)=\gamma u_{4}(x, y, t) \\
& -\frac{1}{2} u_{4}^{2}(x, y, t)-\beta^{2}+\frac{1}{3} \alpha A_{0}-1,
\end{aligned}
$$

where $\xi_{0}$ is an arbitrary integration constant.

As it has already been mentioned in the introduction, it is also possible to apply the achieved solutions of system (2) and (3) to system (1) based on defining the appropriate change of the variable. Therefore, by choosing

$$
u=u(x+y, t), \quad v=v(x+y, t)-1
$$

the solutions of system (2) are the same solutions for system (1). To enclose, it is worth mentioning that the achieved solutions in Section 3.2 can be applied to system (1) in which the similar variable change has properly been defined.

\section{Conclusion}

Two different methods in this study have been employed to result in the exact solutions of the given systems. The transformation of the obtained solutions has been defined in order to gain the exact solutions in which some of them are soliton. Accordingly, exact solutions were obtained to the equations. In spite of the fact that these new solutions may be important for physical problems, this study also suggests that one may find different solutions by choosing different methods. To recapitulate, this method can be utilized to solve many systems of nonlinear partial differential equation arising in the theory of soliton and other related areas of research.

\section{Conflict of Interests}

The authors declare that there is no conflict of interests regarding the publication of this paper.

\section{References}

[1] N. J. Zabusky and M. D. Kruskal, "Interaction of "Solitons" in a collisionless plasma and the recurrence of initial states," Physical Review Letters, vol. 15, no. 6, pp. 240-243, 1965.

[2] R. K. Boullough, "The wave par excellence, the solitary, progressive great wave of equilibrium of the uid an early history of the solitary wave," in Solitons, M. Lak-Shmanan, Ed., Springer Series in Nonlinear Dynamics, pp. 150-281, Springer, Berlin, Germany.

[3] M. R. Miura and E. J. Parkes, Backlund Transformation, Springer, Berlin, Germany, 1978.

[4] V. B. Matveev and M. A. Salle, Darboux Transformations and Solitons, Springer, Berlin, Germany, 1991.

[5] E. J. Parkes and B. R. Duffy, "An automated tanh-function method for finding solitary wave solutions to nonlinear evolution equations," Computer Physics Communications, vol. 98, no. 3, pp. 288-300, 1996.

[6] W. Malfliet and W. Hereman, "The tanh method. I. Exact solutions of nonlinear evolution and wave equations," Physica Scripta, vol. 54, no. 6, pp. 563-568, 1996.

[7] W. Malfliet and W. Hereman, "The tanh method. II. Perturbation technique for conservative systems," Physica Scripta, vol. 54, no. 6, pp. 569-575, 1996.

[8] E. Fan, "Extended tanh-function method and its applications to nonlinear equations," Physics Letters A, vol. 277, no. 4-5, pp. 212-218, 2000.

[9] Y.-T. Gao and B. Tian, "Generalized hyperbolic-function method with computerized symbolic computation to construct the solitonic solutions to nonlinear equations of mathematical physics," Computer Physics Communications, vol. 133, no. 2-3, pp. 158-164, 2001.

[10] X.-Y. Tang and S. -T. Lou, "Localized excitations in $(2+1)$ dimensional systems," Physical Review E, vol. 66, no. 4, pp. 46601-46617, 2002.

[11] M. J. Ablowitz and P. A. Clarkson, Solitons Nonlinear Evolution Equations and Inverse Scattering, vol. 149 of London Mathematical Society Lecture Note Series, Cambridge University Press, New York, NY, USA, 1991.

[12] L. J. F. Broer, "Approximate equations for long water waves," Applied Scientific Research, vol. 31, no. 5, pp. 377-395, 1975.

[13] B. A. Kupershmidt, "Mathematics of dispersive water waves," Communications in Mathematical Physics, vol. 99, no. 1, pp. 5173, 1985.

[14] M. Boiti, J. J. P. Leon, and F. Pempinelli, "Spectral transform for a two spatial dimension extension of the dispersive long wave equation," Inverse Problems, vol. 3, no. 3, article 371, 1987.

[15] B. Tian and Y.-T. Gao, "New families of exact solutions to the integrable dispersive long wave equations in $(2+1)$-dimensional spaces," Journal of Physics A, vol. 29, no. 11, pp. 2895-2903, 1996.

[16] W. Eckhaus, "Preprint 404," Department of Mathematics, University of Utrecht, Utrecht, The Netherlands, 1985. 
[17] T. R. Ding and C. Z. Li, Ordinary Differential Equations, Peking University Press, Peking, China, 1996.

[18] N. Bourbaki, Commutative Algebra, Addison-Wesly, Paris, France, 1971. 


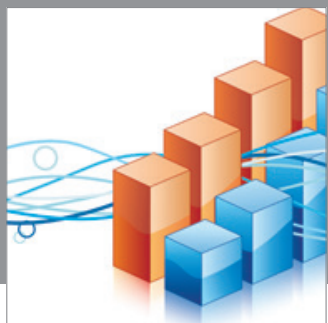

Advances in

Operations Research

mansans

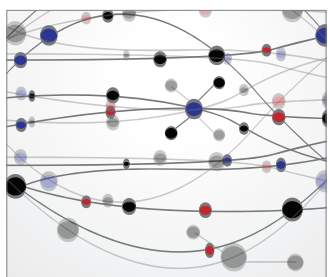

The Scientific World Journal
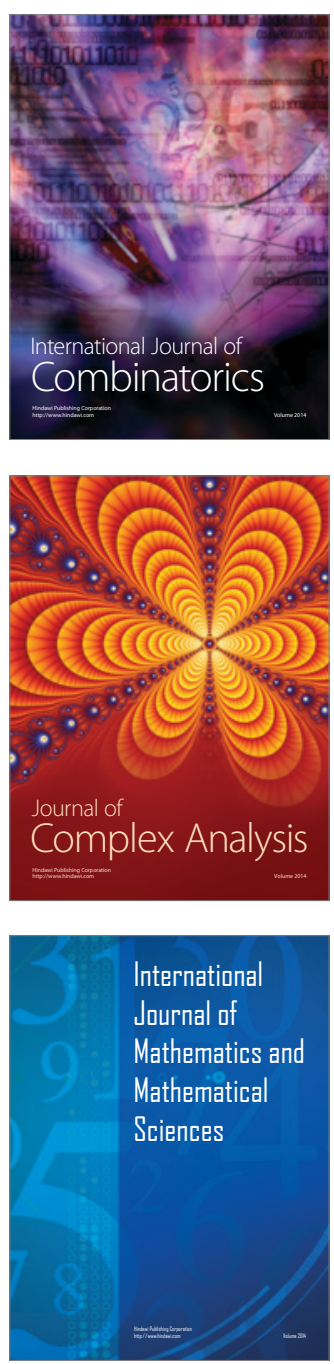
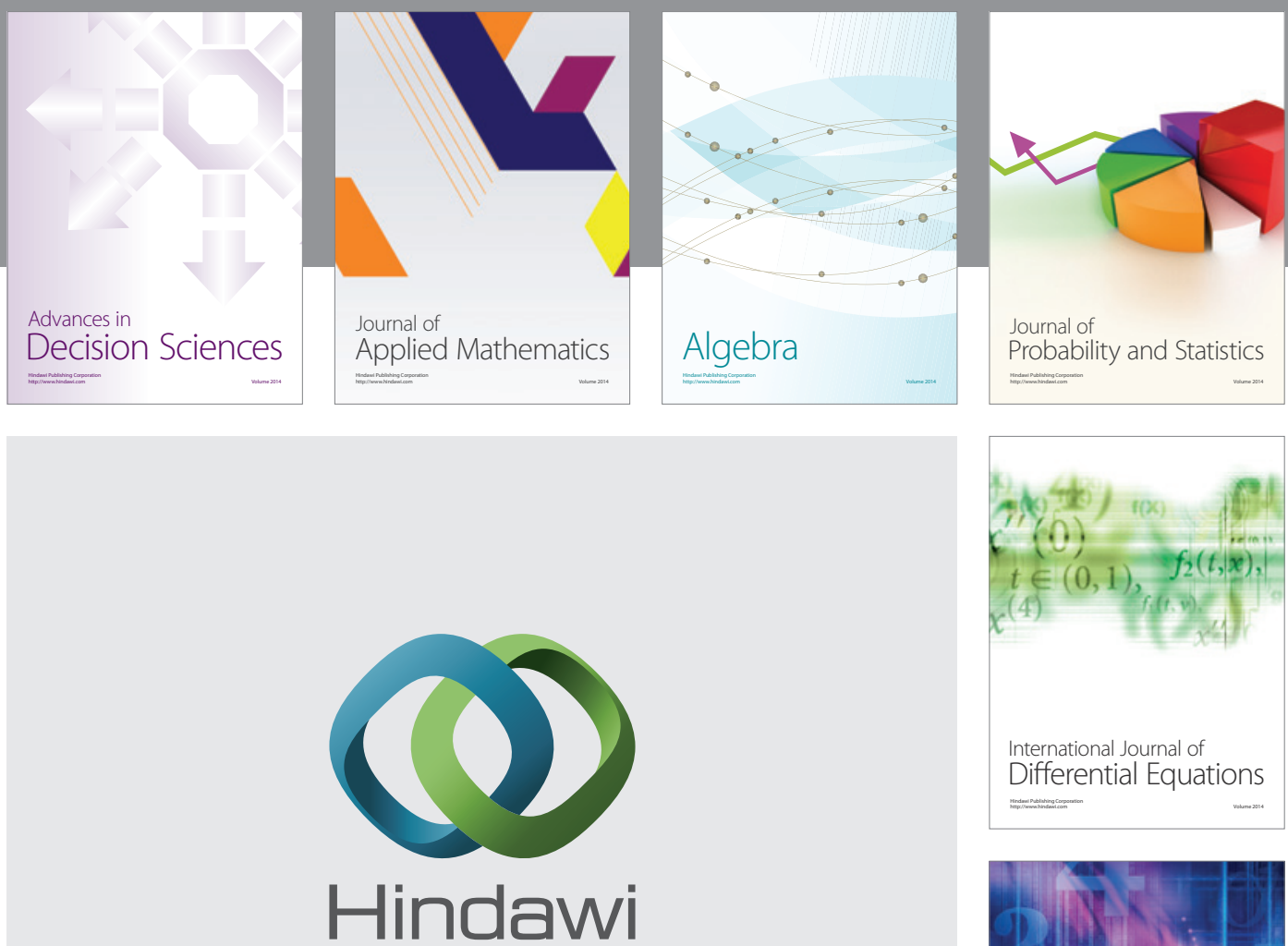

Submit your manuscripts at http://www.hindawi.com
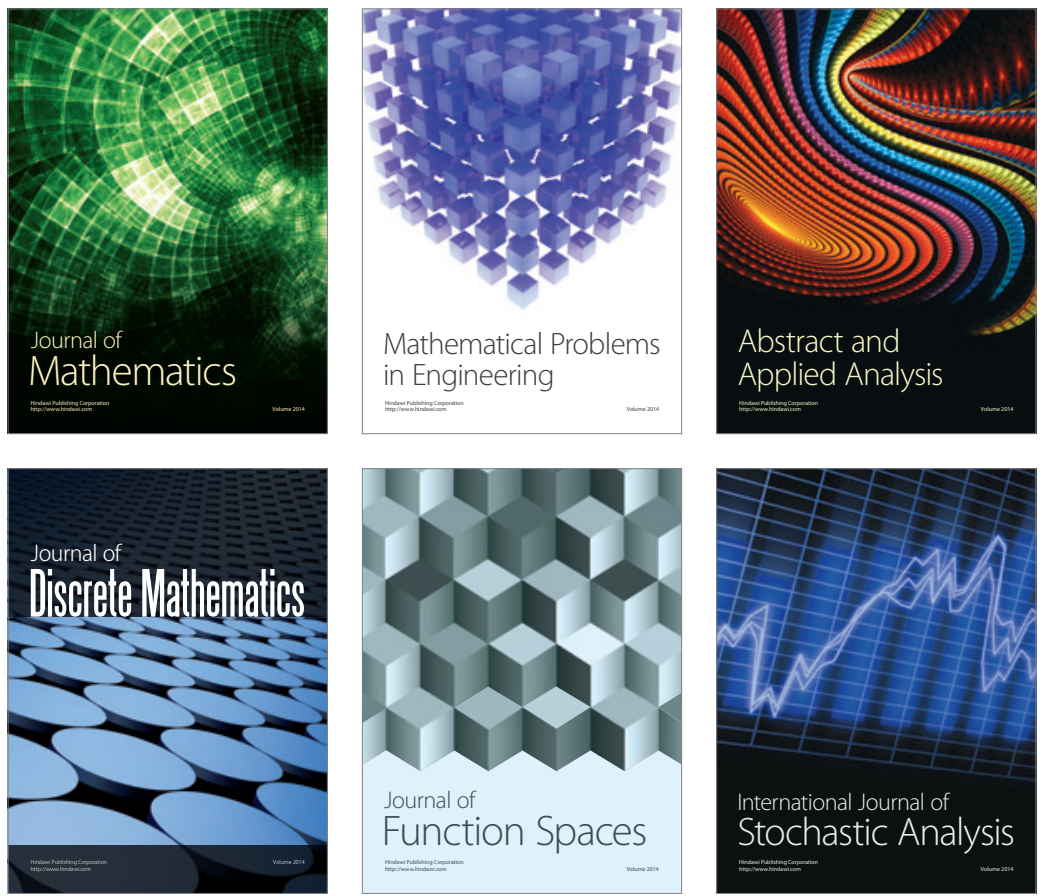

Journal of

Function Spaces

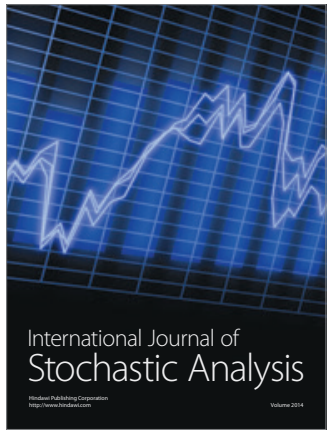

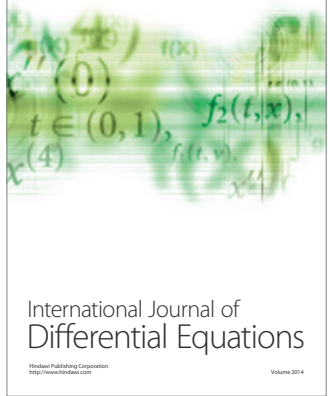
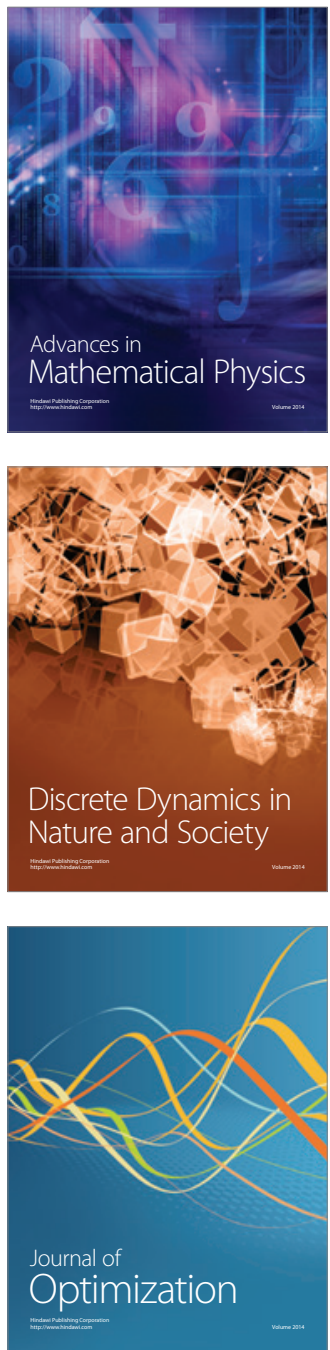